\title{
Análise temporal das tentativas de suicídio atendidas em um hospital de emergência
}

\section{do agreste alagoano, Brasil}

\author{
Temporal analysis of suicide attempts attended at an emergency hospital in agreste Alagoas, Brazil \\ Análisis temporal de intentos de suicidio atendidos en un hospital de urgencias de Alagoas, Brasil
}

Recebido: 04/06/2021 | Revisado: 16/06/2021 | Aceito: 18/06/2021 | Publicado: 25/06/2021

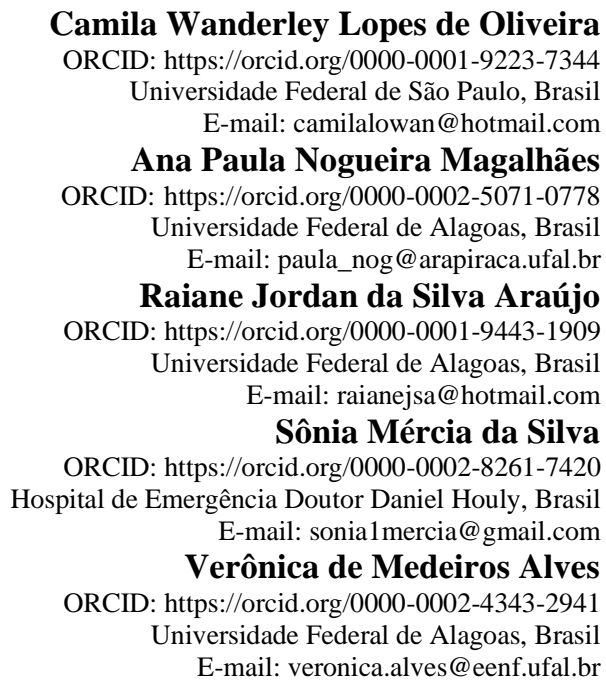

\begin{abstract}
Resumo
Objetivo: Realizar análise temporal das tentativas de suicídio no agreste alagoano. Método: Estudo de abordagem quantitativa com análise documental descritiva, realizado em um hospital de emergência localizado na cidade de Arapiraca, no estado de Alagoas, Brasil. Os dados foram obtidos mediante Ficha de Notificação Individual de Violência Interpessoal e Autoprovocada e Ficha de Notificação de Intoxicação Exógena, relacionadas aos pacientes atendidos por tentativas de suicídio no hospital, entre 2009 e 2017. Resultados: Durante o período de estudo, foi identificado um total de 4.704 atendimentos por tentativas de suicídio. As mulheres $(70,4 \%)$ tentaram mais suicídio que os homens $(29,6 \%)$ e as tentativas foram mais frequentes em adolescentes e adultos jovens entre 15 e 39 anos (75,9\%), com baixa escolaridade $(23,1 \%)$, autodeclarados pardos $(25,9 \%)$ e solteiros $(16,5 \%)$, ocorrendo sobretudo, nos meses de outubro, novembro (ambos com 9,6\%) e dezembro (10,1\%). O método mais utilizado foi a intoxicação exógena por envenenamento $(94,6 \%)$. Houve diferença significativa entre as médias de tentativa de suicídio por mês ao ano ( $\mathrm{p}=$ $0,000)$, faixa etária $(\mathrm{p}=0,023)$ e método de envenenamento $(\mathrm{p}=0,000)$. Conclusão: Entre 2009 e 2017 encontrou-se um número semelhante de casos ao longo dos anos, demonstrando que nenhuma medida preventiva foi implementada pelo serviço de saúde no município. Faz-se necessária a articulação entre a Rede de Atenção Psicossocial para fortalecimento do cuidado na prevenção do suicídio, por meio de intervenção realizada por uma equipe multidisciplinar.
\end{abstract}

Palavras-chave: Tentativa de suicídio; Envenenamento; Epidemiologia.

\begin{abstract}
Objective: To carry out a temporal analysis of suicide attempts in rural Alagoas. Method: Quantitative approach study with descriptive document analysis, carried out in an emergency hospital located in the city of Arapiraca, in the State of Alagoas, Brazil. The data were obtained using the Individual Notification Form for Interpersonal and Self-Harm Violence and the Exogenous Intoxication Notification Form, related to patients treated for suicide attempts in the hospital, between 2009 and 2017. Results: During the study period, a total was identified 4,704 visits for suicide attempts. Women (70.4\%) attempted more suicide than men (29.6\%) and attempts were more frequent in adolescents and young adults between 15 and 39 years old (75.9\%), with low education (23.1\%), self-declared browns $(25.9 \%)$ and single $(16.5 \%)$, occurring mainly in the months of October, November (both with $9.6 \%$ ) and December $(10.1 \%)$. The most used method was exogenous poisoning $(94.6 \%)$. There was a significant difference between the means of suicide attempt per month per year $(\mathrm{p}=0.000)$, age group $(\mathrm{p}=0.023)$ and poisoning method $(\mathrm{p}=0.000)$. Conclusion: Between 2009 and 2017 a similar number of cases was found over the years, demonstrating that no preventive measures were
\end{abstract}


implemented by the health service in the municipality. It is necessary to articulate the Psychosocial Care Network to strengthen care in the prevention of suicide, through an intervention carried out by a multidisciplinary team.

Keywords: Suicide attempted; Poisoning; Epidemiology.

\begin{abstract}
Resumen
Objetivo: Realizar un análisis temporal de los intentos de suicidio en la región rural de Alagoas. Método: Estudio con abordaje cuantitativo con análisis descriptivo de documentos, realizado en un hospital de urgencias ubicado en la ciudad de Arapiraca, en el Estado de Alagoas, Brasil. Los datos se obtuvieron a través del Formulario de Notificación Individual de Violencia Interpersonal y Autoinfligida y el Formulario de Notificación de Intoxicación Exógena, relacionados con pacientes atendidos por intento de suicidio en el hospital, entre 2009 y 2017. Resultados: Durante el período de estudio se identificó un número total . de 4.704 casos de intentos de suicidio. Las mujeres $(70,4 \%)$ intentaron más suicidio que los hombres $(29,6 \%)$ y los intentos fueron más frecuentes en adolescentes y adultos jóvenes entre 15 y 39 años $(75,9 \%)$, con baja escolaridad $(23,1 \%)$, autodeclarada morena $(25,9 \%)$. \%) y único $(16,5 \%)$, ocurriendo principalmente en los meses de octubre, noviembre (ambos con 9,6\%) y diciembre (10,1\%). El método más utilizado fue la intoxicación exógena por intoxicación $(94,6 \%)$. Hubo una diferencia significativa entre las medias de intento de suicidio por mes por año $(\mathrm{p}=0,000)$, grupo de edad $(\mathrm{p}=0,023)$ y método de envenenamiento $(\mathrm{p}=0,000)$. Conclusión: Entre 2009 y 2017 , se encontró una cantidad similar de casos a lo largo de los años, lo que demuestra que el servicio de salud de la ciudad no implementó ninguna medida preventiva. La articulación entre la Red de Atención Psicosocial es necesaria para fortalecer la atención en la prevención del suicidio, a través de una intervención realizada por un equipo multidisciplinar. Palabras clave: Intento de suicidio; Envenenamiento; Epidemiología.
\end{abstract}

\title{
1. Introdução
}

De acordo com a Organização Mundial da Saúde (OMS, 2019), aproximadamente 800.000 pessoas morrem por suicídio todos os anos, tornando-se uma crescente preocupação de saúde pública em todo o mundo. Atualmente, os suicídios ocupam a segunda principal causa de morte no mundo entre jovens de 15 a 29 anos, perdendo apenas para os acidentes de trânsito (WHO, 2008). Aproximadamente 10.000 indivíduos morrem por suicídio a cada ano no Brasil, o que resultou em uma taxa bruta de 5,5 mortes para cada 100.000 habitantes em 2015 (Rodrigues, et al., 2019).

O Sistema de Informação de Agravos de Notificação (SINAN) é um sistema brasileiro cujo objetivo é notificar, investigar, acompanhar e analisar os dados gerados rotineiramente pelo Sistema de Vigilância Epidemiológica (Rocha, et al., 2019). A partir de 2011, a notificação de violência autoprovocada, que compreende autoagressão e tentativa de suicídio, tornouse compulsória no Brasil (Brasil, 2017). Assim, o número de casos registrados cresceu a cada ano, à medida que os profissionais da rede de serviços foram sendo sensibilizados e capacitados para preencherem as fichas de notificação. Esses dados são fundamentais para a geração de informações que possam subsidiar a tomada de decisões e a implementação de políticas públicas.

Entretanto, mesmo com a obrigatoriedade em notificar os casos de tentativas de suicídio, os dados devem ser analisados cuidadosamente, visto que no Brasil ainda há problemas com sub-registros e subnotificações desses casos. Calcula-se que as taxas de tentativas de suicídio podem ser de 10 a 40 vezes maiores, quando comparadas às taxas de suicídio no país (Alves, et al., 2017; Botega, 2014).

Sabe-se que o comportamento suicida é um fenômeno complexo e multifatorial (Brasil, 2019), que envolve questões socioculturais, econômicas, psicológicas, biológicas e ambientais. Portanto, são esperadas variações regionais nas taxas de tentativas de suicídio em um país grande e heterogêneo como o Brasil. Nesse sentido, o presente estudo visa realizar uma análise temporal das tentativas de suicídio na região do agreste alagoano, no período de 2009 a 2017. A análise temporal das tentativas de suicídio é importante porque traz informações que podem ser utilizadas para compreender de que forma acontece este fenômeno.

\section{Metodologia}

Esta pesquisa foi aprovada pelo Comitê de Ética em Pesquisa da Universidade Federal de Alagoas, sob o parecer n. 2.682.215, em 29 de maio de 2018 . 
Este estudo empregou uma abordagem quantitativa com análise documental descritiva, sendo realizado em um hospital de emergência na cidade de Arapiraca, Alagoas, Brasil. A cidade de Arapiraca é a segunda maior do estado de Alagoas e a mais importante do interior, contando com uma população aproximada de 230 mil habitantes (IBGE, 2019). O hospital onde o estudo foi realizado atende exclusivamente por meio do Sistema Único de Saúde (SUS) e é a maior referência no atendimento em urgências e emergências às vítimas de traumas de média e alta complexidade no interior de Alagoas, possuindo uma média anual de 45 mil atendimentos.

Os dados que serviram de base ao estudo foram obtidos mediante os registros de duas fichas de notificação do SINAN: Ficha de Notificação Individual de Violência Interpessoal e Autoprovocada e Ficha de Investigação Individual de Intoxicação Exógena, ambas advindas do registro de pacientes atendidos por tentativa de suicídio, e disponíveis no setor de vigilância epidemiológica hospitalar, no período de primeiro de janeiro de 2009 a 31 de dezembro de 2017.

Selecionaram-se as seguintes variáveis: Faixa etária, sexo, situação conjugal, raça/cor da pele, escolaridade, município e zona de residência, método utilizado, mês de notificação, ano de notificação encaminhamento dos casos e óbito.

Os critérios de inclusão foram todas as Fichas de Investigação Individual de Intoxicação Exógena em que no campo 55 (circunstância da exposição), estavam selecionados apenas o número dez, que se refere aos casos de tentativa de suicídio. Já nas Fichas de Violência Interpessoal e Autoprovocada, foram selecionadas apenas as que tinham a marcação positiva para lesão autoprovocada (campo 54). Os dados levantados foram tabulados em planilha eletrônica e a análise dos dados foi feita por meio do programa SPSS (Statistical Package for Social Sciences) versão 20. Foi realizada uma análise individual das variáveis, por meio da distribuição de frequências e em seguida, foi realizado o teste T de Student, para avaliar as médias das variáveis estudadas, com nível de significância de 5\%.

\section{Resultados}

No período entre 2009 e 2017, foram registrados 4.704 atendimentos por tentativas de suicídio, correspondendo a uma média de 522 episódios por ano. O ano de 2013 foi o que apresentou a maior frequência de casos, seguido de redução até 2016, voltando a uma projeção crescente em 2017. Já a evolução dos óbitos manteve-se de forma retilínea, com ponto de mediana em nove episódios por ano, totalizando 85 (1,8\%) óbitos por tentativas de suicídio nos nove anos avaliados (Figura 1).

Figura 1. Tentativas de suicídio e óbitos por suicídio no Hospital de Emergência do Agreste Alagoano, Arapiraca, Alagoas, Brasil, por ano, 2009-2017.

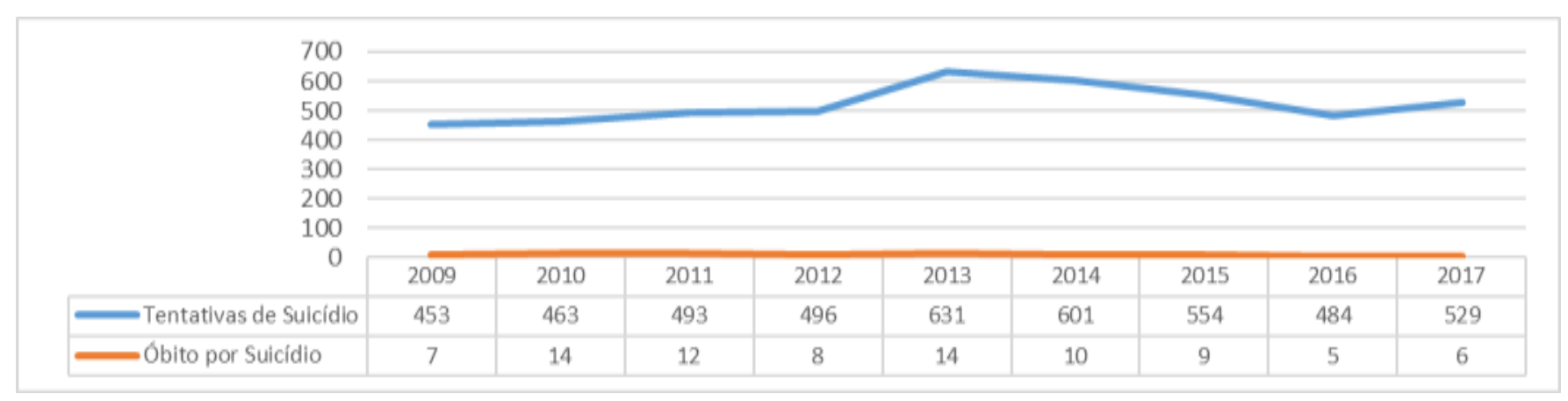

Fonte: SINAN.

Observa-se que as tentativas de suicídio foram predominantes em adultos jovens de 20 a 39 anos (51,7\%), do sexo feminino $(70,4 \%)$, solteiros $(16,5 \%)$, autodeclarados pardos $(25,9 \%)$ e com baixa escolaridade $(23,9 \%)$. Quanto ao método utilizado para a tentativa de suicídio, destaca-se a intoxicação exógena por envenenamento (94,6\%) (Tabela 1). 
Tabela 1. Perfil dos pacientes atendidos por tentativas de suicídio no Hospital de Emergência do Agreste Alagoano, Arapiraca, Alagoas, Brasil, 2009-2017.

\begin{tabular}{|c|c|c|}
\hline CARACTERÍSTICA & $\mathbf{N}$ & $\%$ \\
\hline \multicolumn{3}{|l|}{ Faixa etária } \\
\hline $5-19$ anos & 1465 & $31,1 \%$ \\
\hline 20-39 anos & 2433 & $51,8 \%$ \\
\hline $40-59$ anos & 691 & $14,7 \%$ \\
\hline de 60 acima & 115 & $2,4 \%$ \\
\hline \multicolumn{3}{|l|}{ Sexo } \\
\hline Feminino & 3311 & $70,4 \%$ \\
\hline Masculino & 1393 & $29,6 \%$ \\
\hline \multicolumn{3}{|l|}{ Situação conjugal } \\
\hline Não declarado & 3071 & $65,3 \%$ \\
\hline Solteiro & 776 & $16,5 \%$ \\
\hline Casado / união consensual & 653 & $13,9 \%$ \\
\hline Viúvo & 25 & $0,5 \%$ \\
\hline Separado & 140 & $3,0 \%$ \\
\hline Não se aplica & 39 & $0,8 \%$ \\
\hline \multicolumn{3}{|l|}{ Raça/cor da pele } \\
\hline Não declarado & 2980 & $63,4 \%$ \\
\hline Branca & 384 & $8,2 \%$ \\
\hline Preta & 101 & $2,1 \%$ \\
\hline Amarela & 15 & $0,3 \%$ \\
\hline Parda & 1220 & $25,9 \%$ \\
\hline Indígena & 4 & $0,1 \%$ \\
\hline \multicolumn{3}{|l|}{ Escolaridade* } \\
\hline Não declarado & 3057 & $65,0 \%$ \\
\hline Analfabeto & 147 & $3,1 \%$ \\
\hline $1^{\mathrm{a}}$ a $4^{\mathrm{a}}$ série do $\mathrm{EF}$ & 370 & $7,8 \%$ \\
\hline $5^{\mathrm{a}}$ a $8^{\mathrm{a}}$ série do $\mathrm{EF}$ & 609 & $12,9 \%$ \\
\hline Ensino Médio & 435 & $9,3 \%$ \\
\hline Educação superior & 86 & $1,9 \%$ \\
\hline \multicolumn{3}{|l|}{ Método Utilizado } \\
\hline Espancamento contra o próprio corpo & 20 & $0,4 \%$ \\
\hline Enforcamento & 53 & $1,1 \%$ \\
\hline Objeto contundente ou perfuro-cortante & 123 & $2,7 \%$ \\
\hline Substância ou objeto quente & 5 & $0,1 \%$ \\
\hline Envenenamento & 4451 & $94,6 \%$ \\
\hline Arma de fogo & 25 & $0,5 \%$ \\
\hline Outras agressões e ameaças & 27 & 0,6 \\
\hline
\end{tabular}

*Foi considerado a união dos dados de escolaridade completa e incompleta em cada subitem. Fonte: SINAN.

A maioria (49,4\%) das pessoas que tentaram suicídio residia no município de Arapiraca, sendo 2.795 (59,4\%) residentes na zona urbana da cidade. Após o atendimento e alta hospitalar, mais da metade das pessoas foram encaminhadas para a Atenção Básica de Saúde (55,6\%). Quanto à distribuição sazonal, observa-se que as tentativas de suicídio ocorreram predominantemente na primavera $(1.798-38,2 \%)$ (Figura 2). 
Figura 2. Distribuição dos atendimentos por tentativas de suicídio segundo os meses do ano, no Hospital de Emergência do Agreste Alagoano. Arapiraca, Alagoas, Brasil, 2009-2017.

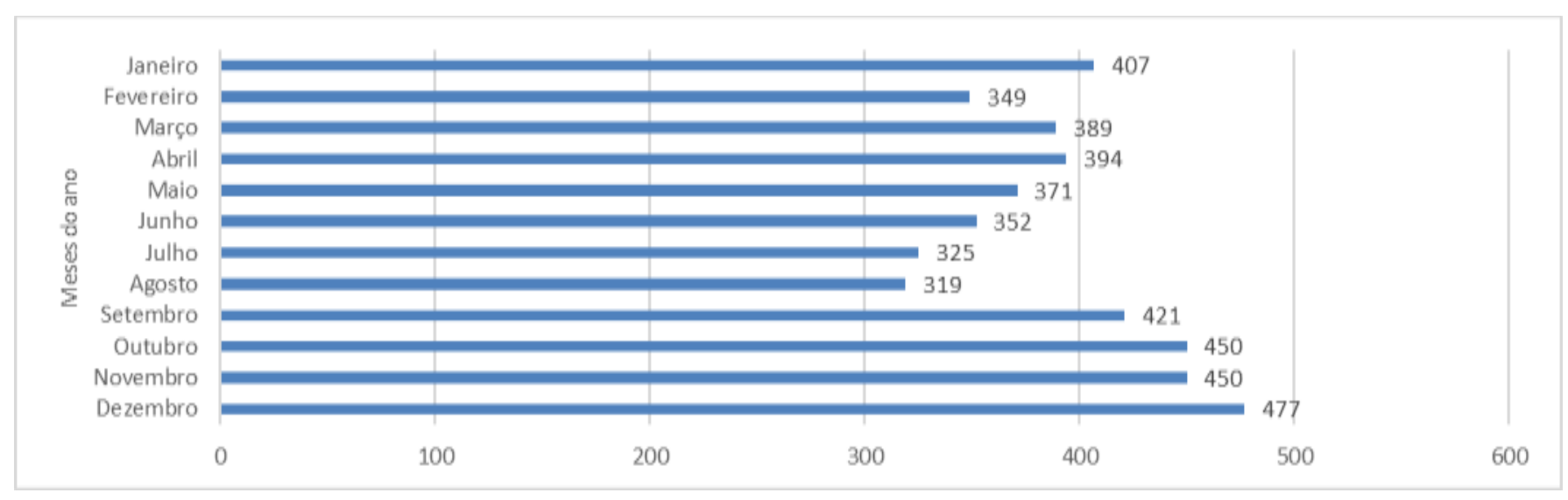

Fonte: SINAN.

Houve diferença estatisticamente significante entre as médias de tentativas de suicídio por mês ao ano (p=0,000), faixa etária $(\mathrm{p}=0,023)$ e método de envenenamento $(\mathrm{p}=0,000)$.

\section{Discussão}

Os resultados obtidos neste estudo indicam que a maioria das vítimas de tentativas de suicídio eram adolescentes e adultos jovens, na faixa etária de 15 a 39 anos, corroborando com a literatura nacional e internacional (Darré, et al., 2019; Xu, Wang, \& Shi, 2018; Bahia, et al., 2017). Os comportamentos suicidas entre adolescentes e adultos jovens envolvem motivações complexas, incluindo abuso sexual, violência, uso de drogas, transtornos mentais, homossexualidade, doença sexualmente transmissível e diminuição de renda (Ribeiro, et al., 2018; Ramôa, et al., 2017).

Os adolescentes, além dos motivos citados acima, podem apresentar dificuldades para se expressarem, impaciência e impulsividade para resolver os problemas que vivenciam. Assim, por meio da tentativa de suicídio, eles podem buscar a atenção da família e da sociedade, como uma forma de pedido de socorro, mostrando que algo está errado. Uma vez que a tentativa de suicídio pode estar presente na adolescência, é importante alertar aos gestores de saúde sobre o risco que essa faixa etária apresenta, para a tomada de medidas que visem à promoção da vida desse grupo populacional. Estudos evidenciam que o suicídio e as tentativas de suicídio afetam significativamente a faixa etária de jovens adultos (Alves, et al., 2017; Moreira, et al., 2015; Brasil, 2007).

Os achados da literatura mostram que as mulheres realizam mais tentativas de suicídio do que os homens (Veloso, et al, 2016; Vieira, Santana \& Suchara, 2015; Brasil, 2007). Os principais fatores que tornam as mulheres mais suscetíveis à ideação e às tentativas de suicídio são: exposição à violência (verbal, psicológica, física, sexual), vulnerabilidade frente a estressores psicossociais e ao desenvolvimento de psicopatologias, além de aspectos culturais, envolvendo uma posição desigual ou falta de poder em relação a seus interesses frente ao sexo oposto (Veloso, et al., 2016).

Sobre a situação conjugal, alguns estudos (Santos, et al., 2017; Nungo, Morales \& Romero, 2017; Vidal, Gotinjo e Lima, 2013) relatam que as pessoas solteiras estão em maior risco de tentar suicídio, corroborando com os achados encontrados nesta pesquisa. Essa relação pode ser parcialmente explicada, considerando que ser solteiro está associado a depressão maior e esquizofrenia, condições psiquiátricas estas que podem aumentar o risco de suicídio (Bando, et al., 2012).

No agreste alagoano, os autodeclarados pardos apresentaram maior número de casos de tentativas de suicídio. Em outros estados brasileiros, a taxa de suicídio também predominou nos autodeclarados pardos (Batista, Araújo \& Figueiredo, 2016; Fernandes, Ferreira \& Castro, 2016). Porém, em um estudo realizado no Município da Região Sul do Brasil, 86,5\% dos casos de 
tentativa de suicídio foram de pessoas autodeclaradas brancas. Isso pode ser explicado pelo fato de que a região foi colonizada por imigrantes vindos da Itália e Alemanha (Ferreira \& Trichês, 2014).

No que diz respeito à escolaridade, é importante destacar que esta variável está fortemente ligada às questões socioeconômicas (Wieczocorkievicz, et al., 2016; Moreira, et al, 2015). Neste estudo, a escolaridade foi uma das variáveis que apresentou porcentagem importante de casos ignorados. Das fichas que tiveram essa característica preenchida, observou-se a maior frequência de baixa escolaridade entre as vítimas de tentativas de suicídio. Dados do Ministério da Saúde (2017) indicam que quanto menor for a escolaridade, maior a incidência de transtornos mentais, inclusive o suicídio. Por outro lado, um nível de escolaridade elevado pode ser um fator protetor para tentativa de suicídio, pois pode contribuir para levar a pessoa a buscar ajuda e a aderir tratamentos (Moreira, et al., 2015).

A intoxicação exógena (medicamentos, agrotóxicos e raticidas) destaca-se por ter sido o método mais utilizado no estudo e está entre as três principais causas de suicídio na população mundial (Woo, et al., 2018; Brasil, 2007). Um dos motivos para a alta frequência de tentativas de suicídio por intoxicações pode ser o fato de os adultos terem fácil acesso a essas substâncias, o que justificaria a escolha desse método no ato suicida. Desse modo, reduzir o acesso às medicações controladas e à agentes tóxicos é uma estratégia de prevenção (Vieira, Santana \& Suchara, 2015). Em estudos anteriores, realizados também no agreste de Alagoas, o envenenamento foi o principal método usado para as tentativas de suicídio. Medicamentos foram os agentes mais frequentes, seguidos pelos venenos (químicos, raticida) (Alves, et al., 2017; Magalhães, et al., 2014).

Ainda sobre o método utilizado para as tentativas de suicídio, aquelas em que foram utilizados objetos cortantes ocuparam o segundo lugar no atual estudo, seguindo o mesmo padrão de outro estudo nacional (Almeida, Crispim \& Peixoto, 2018). Entre os métodos mais agressivos desse estudo, os mais frequentes foram as lesões por armas de fogo auto infligidas, seguidas de enforcamento.

O risco de suicídio aumenta de acordo com o número de tentativas prévias e com o intervalo de tempo menor entre essas tentativas, sendo o primeiro ano o período de maior risco. Dentre os pacientes atendidos em setores de emergência por tentativa de suicídio, foi estimado que $30 \%$ a $60 \%$ tiveram tentativas prévias e que de $10 \%$ a $25 \%$ tentarão novamente no prazo de um ano (Vidal, Gontijo \& Lima, 2013). Neste estudo, não podemos afirmar que houve tentativa prévia de suicídio entre os pacientes atendidos no hospital, devido à falta de registro dessa informação na ficha de notificação. Entretanto, a literatura destaca que para cada tentativa documentada, existem outras quatro que não são registradas (Becker, et al., 2018; Vidal, Gontijo \& Lima, 2013).

Com relação à sazonalidade, algumas pesquisas relataram um maior número de tentativas no outono (Alves et al., 2017; Magalhães, et al., 2014; Vieira, Santana \& Suchara, 2013). No presente estudo, houve maior frequência de tentativas de suicídio na primavera e início do verão (outubro, novembro e dezembro), achado semelhante a estudos epidemiológicos internacionais (Coimbra, et al., 2016; Roehner, 2015). O pico sazonal na primavera para suicídio consumado, acontece particularmente entre indivíduos do sexo masculino, com faixa etária mais elevada e entre aqueles que usam métodos violentos de suicídio (Christodoulou, et al., 2012).

Neste estudo, observou-se a predominância de vítimas de tentativas de suicídio que residiam na zona urbana, o que pode estar associado às condições precárias de trabalho, a baixos salários, e à facilidade para comprarem medicações e venenos. Estas condições de vida podem trazer complicações à saúde mental e física dos indivíduos (Bahia, et al., 2017). Tal achado diverge de estudo anterior (Alves, et al., 2017) realizado na mesma região, e de estudos internacionais, em que o maior número de casos de tentativas de suicídio era de pessoas que viviam na zona rural (Sun \& Zhang, 2018; Goldman-Mellor, Allen \& Kaplan, 2017).

O direcionamento dos casos atendidos por tentativas de suicídio para o serviço de atenção básica levanta a necessidade de se qualificar estes profissionais para acolher esta demanda. A constante qualificação e capacitação dos profissionais da emergência possibilitam à equipe de saúde realizar um atendimento mais eficaz a essas vítimas. Além disso, a interlocução da 
equipe do hospital com os serviços da rede de atenção em saúde mental pode se constituir em estratégia para a construção de um plano de cuidados às vítimas no momento da alta hospitalar (Reisdorfer, 2015).

As principais limitações encontradas neste estudo referem-se ao preenchimento incompleto das fichas de notificação, o que dificultou o acesso a variáveis que são importantes para o entendimento do perfil das vítimas de tentativas de suicídio, como a cor da pele e a escolaridade. A incompletude das informações pode acontecer pelo fato de os profissionais de saúde desconhecerem a importância do preenchimento adequado das fichas de notificação, instrumentos que são fundamentais para o planejamento das ações em saúde e prevenção do comportamento suicida.

\section{Conclusão}

A realização deste estudo permitiu evidenciar a importância do preenchimento completo das fichas de notificação dos atendimentos por tentativas de suicídio, para que assim estes dados possam apontar com maior precisão como se apresenta esse fenômeno nas diferentes regiões do Brasil.

Os dados apresentados são úteis aos gestores de saúde na elaboração de cuidados voltados para a identificação de grupos mais vulneráveis às tentativas de suicídio e seus fatores de risco, sendo necessária maior articulação entre os serviços da Rede de Atenção Psicossocial, para fortalecimento do cuidado na prevenção do suicídio, por meio de intervenções feitas por equipes multiprofissionais.

Sugere-se assim o fortalecimento de ações preventivas mediante a compreensão de que os dados dessa pesquisa indicam a necessidade de efetividade nas ações públicas de prevenção ao suicídio, frente ao número de casos identificados ao longo dos anos estudados.

\section{Referências}

Alves, V. M., Francisco, L. C., Melo, A. R., Novaes, C. R., Belo, F. M., \& Nardi, A. E. (2017). Trends in suicide attempts at an emergency department. Revista Brasileira de Psiquiatria. 39, 55-61.

Almeida, R., Crispim, M. S., \& Peixoto, S. (2018). A prática da automutilação na adolescência: o olhar da psicologia escolar/educacional. Ciências Humanas e Sociais, 4(3), 147-160.

Bahia, C. A., Avanci, J. C., Pinto, L. W., \& Minayo, M. C. S. (2017). Lesão autoprovocada em todos os ciclos da vida: perfil das vítimas em serviços de urgência e emergência de capitais do Brasil. Ciência e saúde coletiva, 22(9):2841-2850.

Bando, D. H., Brunoni, A. R., Fernandes, T. G., Benseñor, I. M., \& Lotufo, P. A. (2012). Suicide rates and trends in Sao Paulo, Brazil, according to gender, age and demographic aspects: a joinpoint regression analysis. Brazilian Journal of Psychiatry, 34(3), $286-293$.

Batista, N. O., Araújo, J. R. C., \& Figueiredo, P. M. (2016). Incidência e perfil epidemiológico de suicídios em crianças e adolescentes ocorridos no estado do Pará, Brasil, no período de 2010 a 2013. Revista Pan-Amazônica de Saúde, 7(4), 61-66.

Becker, S. P., Holdaway, A. S., \& Luebbe, A. M. (2018). Suicidal Behaviors in College Students: Frequency, Sex Differences, and Mental Health Correlates Including Sluggish Cognitive. Journal of Adolescent Health, 63(2), 181-188.

Botega, N. J. (2014). Comportamento suicida: epidemiologia. Psicologia USP. 25(3): 231-236.

Calegaro, V. C., Zatt, C., Bastos, A. G., \& Freitas, L. H. M. (2019) Suicidal patiens in a psychiatric emergency unit: clinical characteristics and aggression profile. Trends in Psychiatry and Psychotherapy, 41(1), 9-17. 10.1590/2237-6089-2017-0149

Christodoulou, C., Douzenis, A., Papadopoulos, F. C., Papadopoulou, A., Bouras, G., Gournellis, R., et al. (2012). Suicide and seasonality. Acta Psychiatrica Scandinavica, 125(2), 127-146.

Coimbra, D. G., Pereira E Silva, A. C., de Sousa-Rodrigues, C. F., Barbosa, F. T., de Siqueira Figueredo, D., Araújo Santos, J. L., Barbosa, M. R., de Medeiros Alves, V., Nardi, A. E., \& de Andrade, T. G. (2016). Do suicide attempts occur more frequently in the spring too? A systematic review and rhythmic analysis. Journal of affective disorders, 196, 125-137.

Darré, T., Consuela, K. A. C., Saka, B., Djiwa, T., Ekouévi, K. D., \& Napo-Koura, G. (2019). Suicidal ideation and suicide attempts in subjects aged 15-19 in Lomé (Togo). BMC Research Note, 12, 187.

Fernandes, D. A. A., Ferreira, N. S., \& Castro, J. G. D. (2016) Perfil epidemiológico das tentativas de suicídio em Palmas-Tocantins, de 2010 a 2014. Tempus Actas de Saúde Coletiva, 10(4), 09-23. 
Ferreira, V. R. T., \& Trichês, V. J. S. (2014). Epidemiological profile of suicide attempts and deaths in a southern Brazilian city. Psico, 45(2), $219-27$.

Goldman-Mellor, S., Allen, K., \& Kaplan, M. S. (2017). Rural/Urban Disparities in Adolescent Nonfatal Suicidal Ideation and Suicide Attempt: A PopulationBased Study. Suicide and Life-Threatening Behavior, 48(6),709-719.

Instituto Brasileiro de Geografia e Estatística (IBGE). (2019). Panorama, Arapiraca.

Magalhães, A. P. N., Alves, V. M., Comassetto, I., Lima, P. C. Faro, A. C. M., \& Nardi, A. E. (2014). Atendimento a tentativas de suicídio por serviço de atenção pré-hospitalar. Jornal Brasileiro de Psiquiatria, 63(1), 16-22.

Moreira, D. L., Martins, M. C., Gubert, F. A., \& Sousa, F. S. P. (2015). Perfil de pacientes atendidos por tentativa de suicídio em um centro de assistência toxicológica. Ciencia y enfermeria, XXI(2), 63-75.

Ministério da Saúde. Secretaria de Vigilância em Saúde. (2019). Perfil epidemiológico dos casos notificados de violência autoprovocada e óbitos por suicídio entre jovens de 15 a 29 anos no Brasil, 2011 a 2018. Boletim Epidemiológico, 50(24), Brasília: Ministério da Saúde. https://portalarquivos2.saude.gov.br/ images/pdf/2019/setembro/13/BE-suic--dio-24-final.pdf.

Ministério da Saúde. Secretaria de Vigilância em Saúde. (2017). Perfil epidemiológico das tentativas e óbitos por suicídio no brasil e a rede de atenção à saúde. Boletim Epidemiológico, 48(30), 1-14. http://portalarquivos2. saude.gov.br/images/pdf/2017/setembro/21/ 2017-025-Perfil-epidemiologico-das-tentativas-eobitos-por-suicidio- -no-Brasil-e-a-rede-de-atencao-a-saude. pdf.

Ministério da Saúde. (2017). Consolidação das normas sobre os sistemas e os subsistemas do Sistema Único de Saúde. Portaria de Consolidação GM/MS nº 04, de 28 de setembro 2017. Diário Oficial [da] República Federativa do Brasil, Brasília, DF, de 03 de outubro de 2017 b.

Ministério da Saúde. Secretária de vigilância em Saúde. Suicídio: tentativas e óbitos por intoxicação exógena no Brasil.

Nungo, L. F. A., Morales, M. A. S., \& Romero, H. G. (2017). El intento de suicidio en Ibagué: el silencio de una voz de auxilio. Revista Criminalidad, 59(2), $81-92$.

Ramôa, A. F. A. S., Soares, C. C. J., Sequeira, J. F. N., \& Azenha, S. (2017). Suicidical behaviour: characterisation and discussion of vulnerability factors. Ver Port Med Geral Fam, 33(5), 321-322.

Reisdorfer, N., Araujo, G. M., Hildebrandt, L. M., Gewehr, T. R., Nardino, J., \& Leite, M. T. (2015). Suicídio na voz de profissionais de enfermagem e estratégias de intervenção diante do comportamento suicida. Revista de Enfermagem da UFSM, 5(2), 295-304.

Roehner, B. M. (2015). How can one explain changes in the monthly pattern of suicide? Physica A: Statistical Mechanics and its Applications, 424, 350-362. 10.1016/j.physa.2015.01.017

Ribeiro, N., Castro, S. S., Scatena, L. M., \& Hass, V. J. (2018). Análise da tendência temporal do suicídio e de sistemas de informações em saúde em relação às tentativas de suicídio. Texto e Contexto Enfermagem, 27(2), e2110016.

Rodrigues, C. D., Souza, D. S., Rodrigues, H. M., \& Konstantyner, T. C. R. O. (2019). Trends in suicide rates in Brazil from 1997 to 2015. Brazilian Journal of Psychiatry, 41(5),380-388.

Rocha, M. S., Oliveira, G. P., Guillen, L. C. T., Coeli, C. M., Saraceni, V., \& Pinheiro, R. S. (2019). Uso de linkage entre diferentes bases de dados para qualificação de variáveis do Sinan-TB e a partir de regras de scripting. Cadernos de Saúde Pública, 35(12), e0007431.

Santos, M. S. P., Santos, M. S. P., Silva, T. P. S., Pires, C. M. C., Ramos, P. G. X., \& Sougey, E. B. (2017). Identificação de aspectos associados à tentativa de suicídio por envenenamento. Jornal Brasileiro de Psiquiatria, 66(4), 197-202.

Silva, L. L. T., \& Madeira, A. M. F. (2014). Tentativa de autoextermínio entre adolescentes e jovens: uma análise compreensiva. Revista de Enfermagem do Centro Oeste Mineiro, 3(4):1281-9.

Sun, L., \& Zhang, J. (2018). Suicide Acceptability and Suicide Attempt. The Journal of Nervous and Mental Disease, 206(9), 694-698.

Vieira, L. P., Santana, V. T. P., \& Suchara, E. A. (2015). Caracterização de tentativas de suicídios por substâncias exógenas. Cadernos de Saúde Coletiva, 23(2), $118-123$.

Veloso, C., Monteiro, L. S. S., Veloso, L. U. P., Moreira, I. C. C., \& Monteiro, C. F. S. (2016). Suicide attempts cared for by a mobile emergency pre-hospital care service. Revista de Enfermagem da UFPI, 5(3), 48-53.

Vidal, C. E. L., Gontijo, E. C. D. M., \& Lima, L. A. (2013). Tentativas de suicídio: fatores prognósticos e estimativa do excesso de mortalidade. Cadernos de Saúde Pública, 29(1), 175-187.

Xu, Y., Wang, C., \& Shi, M. (2018). Identifying Chinese adolescents with a high suicide attempt risk. Psychiatry Research, $269,474-480$.

World Health Organization. Suicide. (2019). https://www.who.int/news/item/09-09-2019-suicide-one-person-dies-every-40-seconds

World Health Organization. (2008). Preventing Suicide A Resource for Media Professionals. Department of Mental Health and Substance Abuse.

Wieczocorkievicz, A. M., Mazon, L. M., Raissa, C. M., Maia, E. W., \& Siebeneichler, J. L. (2016). Caracterização das tentativas de suicídio atendidas em pronto atendimento geral. Saúde em Revista, 16(43), 53-62. 10.15600/2238-1244/sr.v16n43p53-62.

Woo, S., Lee, S. W., Lee, K., Seo, W. S., Lee, J., Kim, H. C., \& Won, S. (2018). Characteristics of High-Intent Suicide Attempters Admitted to Emergency Departments. Journal of Korean Medical Science, 33(41), e259 